\title{
Calibration of Projector Lens Distortions for Quantitative High-Resolution TEM
}

\author{
F. Hue*, C.L. Johnson**, S. Lartigue-Korinek*, G. Wang*, P.R. Buseck***,****, and M.J. Hÿtch* \\ * Centre d'Etudes de Chimie Métallurgique, CNRS, 15 rue G. Urbain, 94407 Vitry-sur-Seine, \\ France \\ ** Center for Functional Nanomaterials, Brookhaven National Lab, Bldg. 480, Upton, NY 11973 \\ *** Dept. of Chemistry \& Biochemistry, Arizona State University, Tempe, AZ, 85287 \\ **** Dept. of Geological Sciences, Arizona State University, Tempe, AZ, 85287
}

Strain mapping by geometric phase analysis (GPA) of high-resolution transmission electron microscope (HRTEM) images has emerged as a powerful technique for materials science [1-3]. Improving the accuracy of GPA requires detailed knowledge of the effects of the microscope on the local lattice information transferred to the HRTEM image. While artifacts resulting from the objective lens transfer have been thoroughly investigated, the large-scale geometrical distortions of HRTEM images caused by the projector lens system have received less attention. However, many materials science problems require measurements of strain over large areas $(>50 \mathrm{~nm})$, where largescale distortions have important effects.

We measured these distortions for various electron microscopes and detection methods (film, image plate, and CCD camera) by analyzing HRTEM images of perfectly crystalline Si (Fig. 1a), and compared our results with electron optical theory [4]. The distortions result in an apparent strain, which was characterized by measuring the local displacements by GPA (Figs. 1b,c). Numerical differentiation of these displacements gives the local magnification and rotation, which are analogous to local dilatation and rigid-body rotation, respectively. Our results show that magnification can vary by as much as $5 \%$ and rotation by $2^{\circ}$ across the field of view of a typical TEM negative (Figs. 2a,b). As such, the distortions can constitute a significant source of error for quantitative displacement and strain mapping, as well as for local lattice parameter determination.

Accurate mapping the medium- and long-range strain fields of various 2- and 3-dimensional defects and local lattice spacings, therefore necessitates removal of these distortions. By subtracting the apparent displacement field caused by the distortions from experimental images, we were able to quantify strains to $0.1 \%$ and rotation to $0.1^{\circ}$ with nanometer spatial resolution. This method of calibration must be applied to individual microscopes since each exhibits a different degree of distortion. Fortunately, our experiments suggest that the projector lens distortions are quite stable over time, as shown by successful correction of images obtained as many as 4 years prior to the calibration images. Finally, the means of image digitization also affects the results. Both flat-bed and drum scanners introduce high-frequency distortions that result from mechanical instabilities in the movement of the CCD bar or negative holder. We have found that image plates and CCD cameras exhibit no such distortions.

[1] M.J. Hÿtch et al. Ultramicroscopy 74 (1998) 131.

[2] M.J. Hÿtch et al. Nature 423 (2003) 270.

[3] C.L. Johnson et al. Proc. Natl. Acad. Sci. USA 101 (2004) 17936.

[4] P.W. Hawkes and E. Kasper, Principles of Electron Optics: Vol. 1. Acad. Press, London (1996).

[5] Partial financial support (for CLJ and PRB) was provided by National Science Foundation grant

EAR-0003533. 


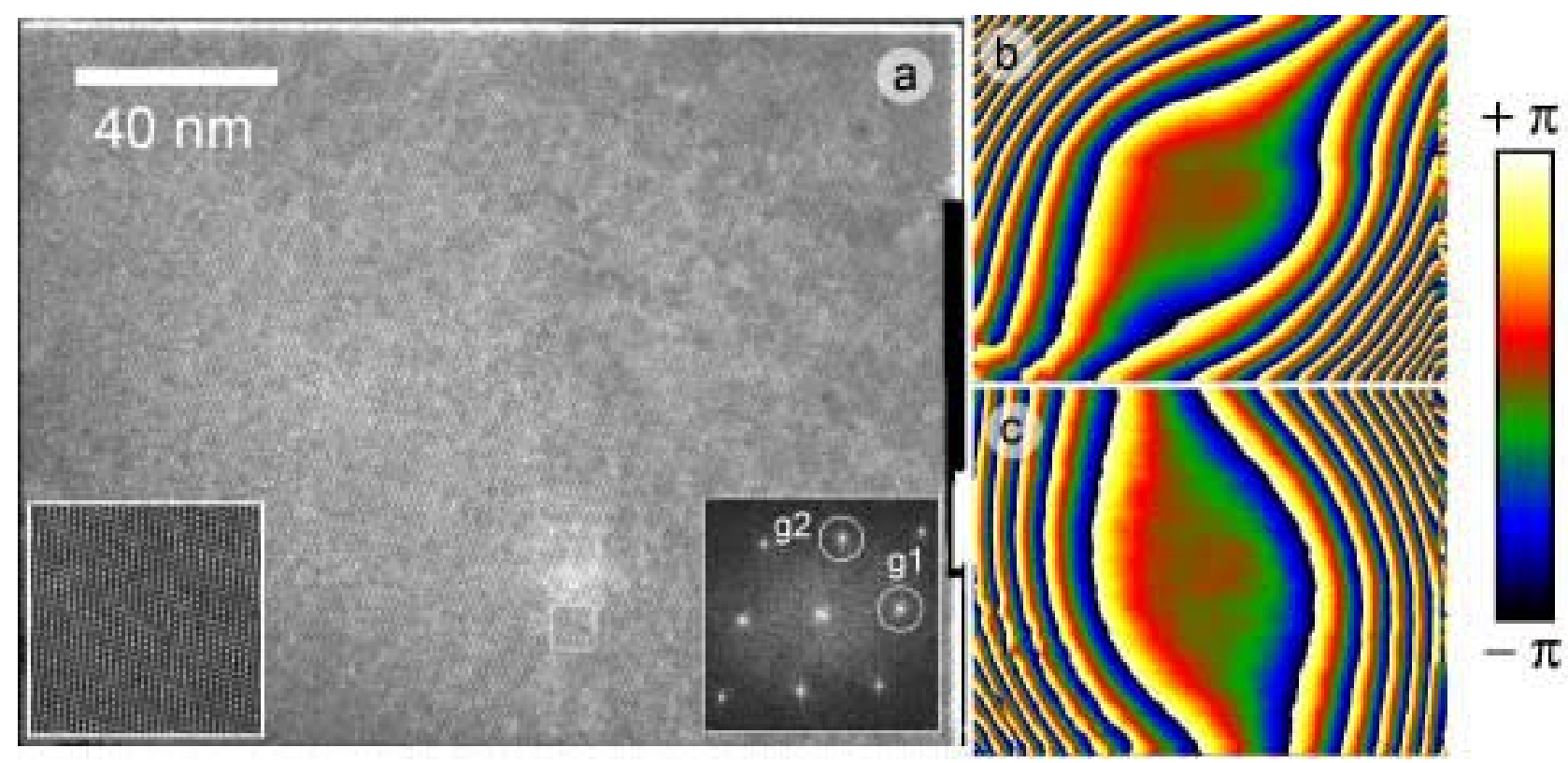

Figure 1. Phase analysis of perfectly crystalline Si using a JEOL 4000EX. (a) HRTEM image of Si taken at $382 \mathrm{KX}$ magnification on a film negative. The boxed region is shown enlarged on the left and the digital diffractogram is inset on the right. (b) Geometric phase image for $\mathrm{g} 1=11-1$ lattice fringes and (c) g2 $=111$ fringes. The color range is from $-\pi$ to $+\pi$ which is proportional to the apparent displacement field caused by the projector lens.
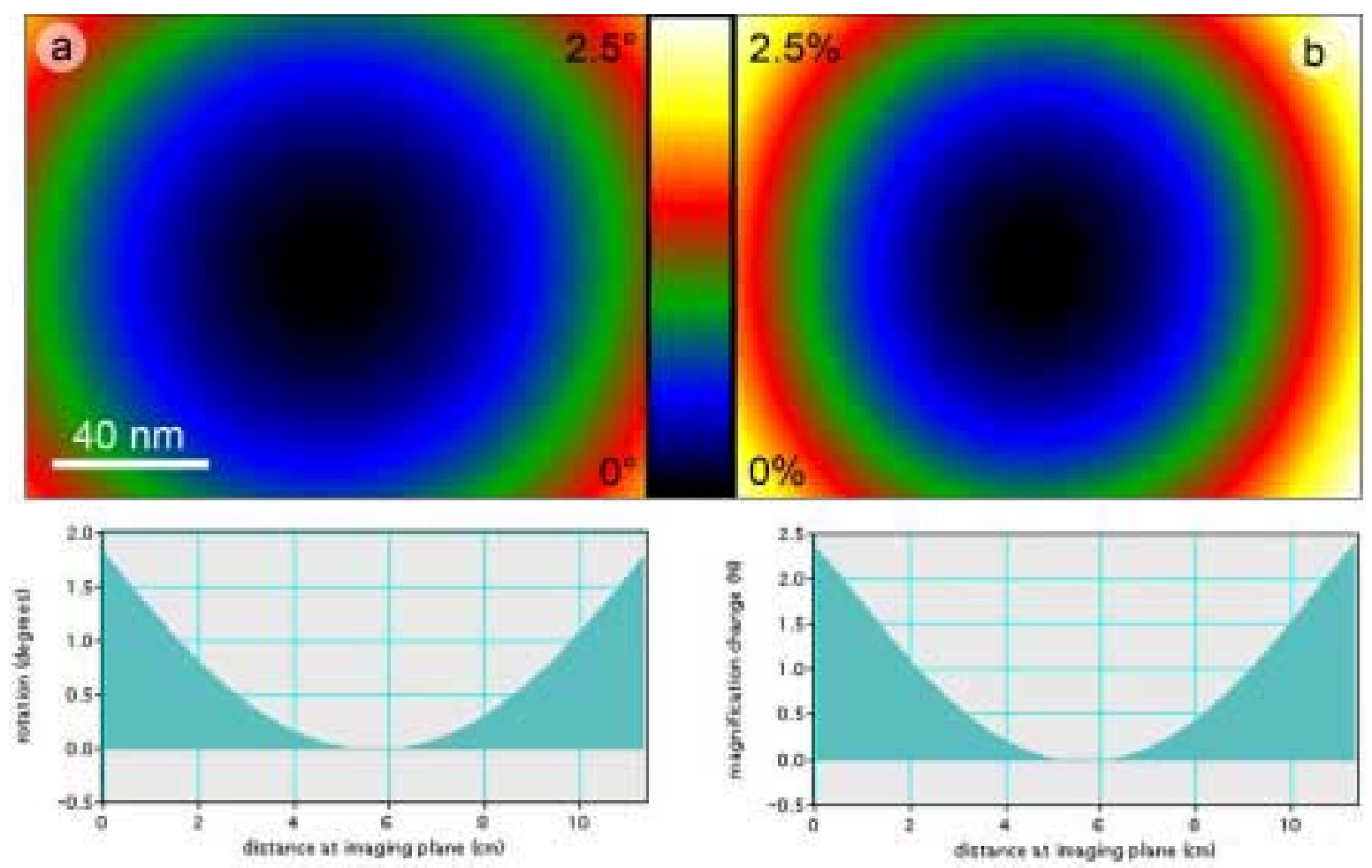

Figure 2. Projector lens distortions determined using Figs $1 \mathrm{~b}$ and $1 \mathrm{c}$ above fitted to a $3^{\text {rd }}$ order polynomial. (a) Spiral distortion (anti-clockwise rotation) and (b) pincushion distortion (magnification change) along with profile plots along the diagonal from upper left to lower right. 